\title{
Design of Crane System Based on an Improved Active Disturbance Rejection Controller
}

\author{
Jie Zhang ${ }^{1,2, a}$, Zhenye Dong ${ }^{1,2}$ \\ ${ }^{1}$ School of Automation, Beijing Institute of Technology, Beijing 100081, China \\ ${ }^{2}$ State Key Laboratory of Intelligent Control and Decision of Complex Systems, Beijing 100081, \\ China \\ a'Zhangjie9107@sina.com
}

\begin{abstract}
Keywords: Crane, Under-actuated System, Robustness, ADRC, Feedforward.
Abstract. An improved ADRC controller is designed for the crane system. Firstly, the extended state observer (ESO) is used to observe the total system disturbance consisting of the system's dynamic perturbation, the system model variations and outside disturbance such as the wind effect. Secondly, to eliminate the observed disturbances. A proportional controller is used to eliminate sway angle of the load, while a feedforward controller is used to improve the dynamic performance of the system. The proposed method has been verified through simulation test.
\end{abstract}

\section{Introduction}

Crane is a kind of typically non-linear, strongly coupled and under-actuated system. It has been widely applied to many areas of industrial production and port transportation. However, it is usually accompanied by a non-complete constraint, which has brought great challenges to the system control.

Targets of crane control system can be addressed as following two points: firstly, the trolley should be controlled to reach the precise position quickly in order to deliver the load efficiently; secondly, the load swing must be suppressed as small as possible in order to avoid collision with surrounding cargo or personnel. Control methods for crane system can be divided into two categories: the open-loop control and the closed-loop control, such as input shaping [1], feedforward control [2], fuzzy control [3], sliding mode control [4], etc. Most of these control methods require high model accuracy. However it is difficult to obtain an accurate model. Closed-loop control method can be applied to reduce the deviation and it is less insensitive to external disturbances and changes in system parameters. But in general, the calculation with closed-loop controller is more complex and the parameters are more difficult to adjust. ADRC is a novel controller with such ability that the external disturbances and internal model parameter uncertainty of the system can be viewed as a general disturbance and be followed by extended state observer (ESO), and be cancelled in control law [5]. Therefore, the strict precise model of the system is not needed in ADRC control. In this paper, an improved ADRC with a proportional controller and a feedforward controller is designed to meet the demands of the crane control.

\section{System Modeling}

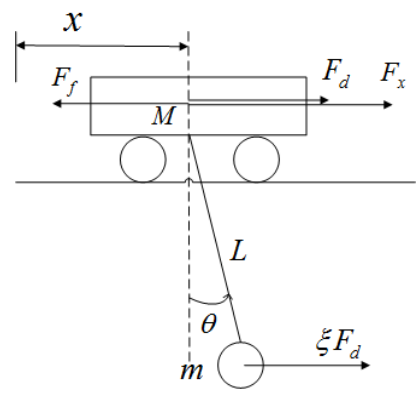

Fig. 1: Model of crane control system 
From the simplified model [6], Euler Lagrange equation is used in two-dimensional overhead crane system modeling. The system model can be expressed:

$$
\begin{aligned}
& F_{x}+F_{d}=D \dot{x}+(M+m) \ddot{x}+m L\left(\ddot{\theta} \cos \theta-\dot{\theta}^{2} \sin \theta\right)+2 m \dot{L} \dot{\theta} \cos \theta+m \ddot{L} \sin \theta . \\
& \frac{\cos \theta}{m} \xi F_{d}=L \ddot{\theta}+2 \dot{L} \dot{\theta}+\ddot{x} \cos \theta+g \sin \theta .
\end{aligned}
$$

Where, $M$ and $m$ denote the mass of the crane $(\mathrm{kg})$ and the load $(\mathrm{kg})$ respectively; $g$ represents the acceleration of gravity $\left(\mathrm{m} / \mathrm{s}^{2}\right) ; D$ represents the friction coefficient between the trolley and the rail; $F_{x}$ represents the tractive force of the trolley $(N)$ and $F_{d}$ represents the wind disturbances in the horizontal direction $(N) ; x(t), L(t), \theta(t)$ stand for the horizontal displacement of the trolley, rope length and load's swing angle to the vertical direction respectively. $\xi$ is the ratio between the load and the trolley's windward area.

Since the swing angle is small during the movement under control, therefore $\theta \approx 0$, $\sin \theta \approx \theta, \dot{\theta} \sin \theta \approx 0$. The crane's rope length changes little during the process, so we can assume $\dot{L} \approx \ddot{L} \approx 0$ [7]. With no consideration of wind disturbance, the simplified linearized mathematical model can be obtained:

$$
\begin{aligned}
& x:(M+m) \ddot{x}+\mathrm{D} \dot{x}+m L \ddot{\theta}=F_{x} . \\
& \theta: L \ddot{\theta}+\ddot{x}+g \theta=0 .
\end{aligned}
$$

Obtained by Eq.3 and Eq.4:

$$
\begin{aligned}
& G_{1}(s)=\frac{\theta(s)}{F_{x}(s)}=\frac{-s}{M L s^{3}+D L s^{2}+(M+m) g s+D g} . \\
& G_{2}(s)=\frac{x(s)}{\theta(s)}=\frac{-L s^{2}-g}{s^{2}} .
\end{aligned}
$$

In this paper, the position of the load can be obtained by $x+L \sin \theta \approx x+L \theta$.

The drive is selected to work in the current loop mode. The system block diagram is as Fig.2.

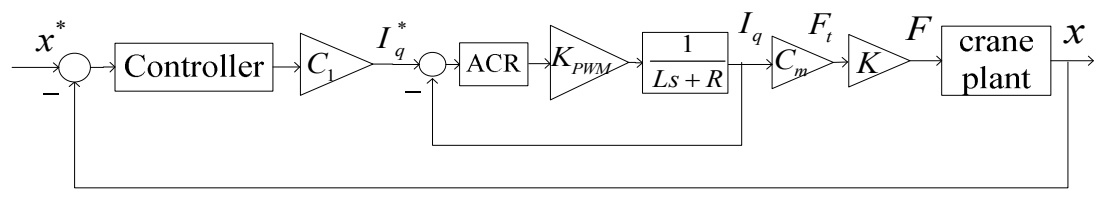

Fig. 2: Block diagram when drive works in the current loop

The controller of the system is the position loop controller to be designed; ACR is the current loop controller which is often a PI controller; $K_{P W M}$ is the drive amplifier gain; $\mathrm{R}$ is the DC motor armature resistance which is $0.41 \Omega$; $\mathrm{L}$ is the DC motor armature inductance which is $0.00411 \mathrm{H} ; x^{*}$ represents the given position; $C_{m}$ delegate the torque constant; $K$ is the ratio between the motor torque and straight thrust; $C_{1}$ is the ratio between controller outputs and the current which is $1 / C_{m} K_{t}$. $F_{t}$ stands for the torque; $F$ stands for the straight thrust acting on the plant.

While $\mathrm{L}$ is much smaller than $\mathrm{R}$, so we can ignore the inductance. The transfer function of the current loop can be described as:

$$
\frac{K_{P W M} K_{p} R s+K_{P W M} K_{I} R}{\left(K_{P W M} K_{p} R+R\right) s+K_{P W M} K_{I} R}
$$

While $\mathrm{R}$ is much smaller than $K_{P W M} K_{p} R$, the motor and drive dynamics can be ignored.

\section{Controller Design And Stability Analysis}

In this paper, we will compare the proposed solution with an existing solution. In the existing solution, position of the trolley and angle of the load are controlled by a PID controller respectively.

The designed controller contains five parts, they are transient process, PD controller, extended state observer, error compensation control rate and a proportional controller. It is designed as Fig.3. 


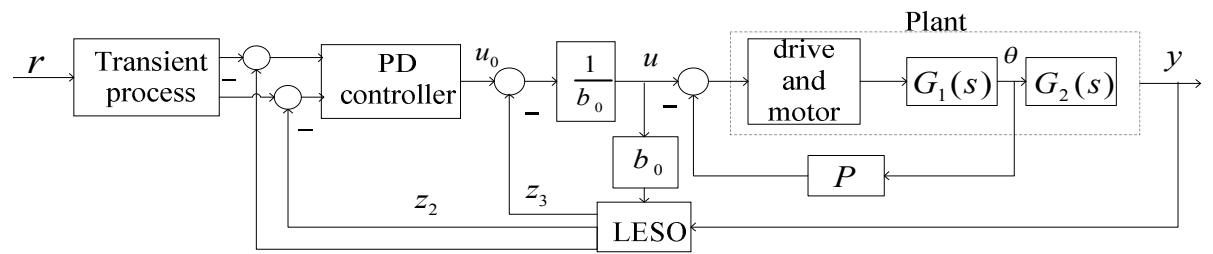

Fig. 3: Block diagram of the ADRC controller

Rewrite the position of the trolley $x$ as $y$, rewrite the control command $F_{x}$ as $u$. Noticing from Eq.5 and Eq. 6 that when $m$ approaches 0 , the system will have two conjugate poles very close to the imaginary axis and that will introduce oscillation. So an angle feedback controller is designed to keep the conjugate poles away from the imaginary axis and make the angle decay fastly. A proportional controller is chosen as the angle feedback controller. The controlled object with the proportional controller is considered as a whole, the transfer function can be obtained:

$$
\frac{y(s)}{u(s)}=\frac{L s^{2}+g}{M L s^{4}+D L s^{3}+\left((M+m) g-k_{p}\right) s^{2}+D g s} .
$$

In order to design an ADRC controller, the equation can be rewritten as:

$$
\ddot{y}=\frac{g}{(M+m) g-k_{p}} u+\frac{L}{(M+m) g-k_{p}} \ddot{u}-\frac{M L}{(M+m) g-k_{p}} \dddot{y}-\frac{D L}{(M+m) g-k_{p}} \dddot{y}-\frac{D g}{(M+m) g-k_{p}} \dot{y} .
$$

Among them:

$$
\begin{aligned}
& b_{0}=\frac{g}{(M+m) g-k_{p}} . \\
& f=\frac{L}{(M+m) g-k_{p}} \ddot{u}-\frac{M L}{(M+m) g-k_{p}} \dddot{y}-\frac{D L}{(M+m) g-k_{p}} \dddot{y}-\frac{D g}{(M+m) g-k_{p}} \dot{y}+w .
\end{aligned}
$$

$w$ is the external disturbance and $f$ is the total disturbance.

Designing the ESO as follows:

$$
\left\{\begin{array}{l}
e=z_{1}-y \\
\dot{z}_{1}=z_{2}-\beta_{1} e \\
\dot{z}_{2}=z_{3}-\beta_{2} e+b_{0} u \\
\dot{z}_{3}=-\beta_{3} e
\end{array}\right.
$$

Where, $z_{1}, z_{2}, z_{3}$ are observer's estimated value $y, \dot{y}, f$ respectively; $e$ is the observer error; $\beta_{1}, \beta_{2}, \beta_{3}$ are gains of the observer. Characteristic equation of the observer is:

$s^{3}+\beta_{1} s^{2}+\beta_{2} s+\beta_{3}=0$.

Configuring observer's all poles at $-\omega_{0}$, we can get: $\beta_{1}=3 \omega_{o}, \beta_{2}=3 \omega_{o}^{2}, \beta_{3}=\omega_{o}^{3}$.

Control law is:

$u=\frac{u_{0}-z_{3}}{b_{0}}$.

With ESO and the control law, the system is deformed into cascaded integrators:

$\ddot{y}=b_{0} u+f \approx u_{0}$.

The second order integral plant can be well controlled with a PD controller:

$u_{0}=k_{p}\left(r-z_{1}\right)+k_{d}\left(-z_{2}\right)$.

Configuring the closed loop poles at $-\omega_{c}$, we can get: $k_{p}=\omega_{c}^{2}, k_{d}=2 \omega_{c}$.

Firstly we must guarantee the stability of the system, in this context we select: $\omega_{0}=15, \omega_{c}=2$.

Table 1: Look up table

\begin{tabular}{|c|c|c|c|c|c|}
\hline Breakpoints & 0 & 0.667 & 1.334 & 2 & 4 \\
\hline Table data & 0 & 0.75 & 0.75 & 0 & 0 \\
\hline
\end{tabular}


In this paper, a look-up table module is used to make a ramp signal convert into a trapezoid signal, its output is integrated to obtain a smoothed S-sized input signal for the system. The lookup table is set as Table 1 .

To analyze the stability of the system in the frequency domain, the system equations is simplified to a feedback form [8], and it is shown in Fig.4.

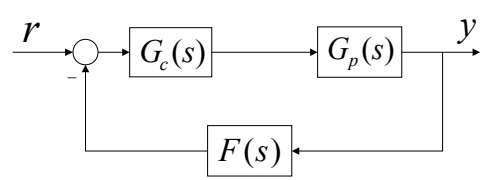

Fig.4: ADRC transfer function block diagram

Eq.12 can be transformed into:

$$
\left\{\begin{array}{l}
\dot{z}_{1}=z_{2}+\beta_{1}\left(y-z_{1}\right) \\
\dot{z}_{2}=z_{3}+\beta_{2}\left(y-z_{1}\right)+b_{0} u \\
\dot{z}_{3}=\beta_{3}\left(y-z_{1}\right)
\end{array}\right.
$$

We can get expressions of $z_{1}, z_{2}, z_{3}$ through the above expression and then put them into Eq.14 and Eq.16, the closed loop transfer function of the system can be obtained:

$$
G=\frac{G_{c} G_{p}}{1+G_{c} G_{p} F} .
$$

Where:

$$
\begin{aligned}
& G_{c}(s)=\frac{k_{p}\left(s^{3}+\beta_{1} s^{2}+\beta_{2} s+\beta_{3}\right)}{b_{0}\left\{s^{3}+\left(\beta_{1}+k_{d}\right) s^{2}+\left(\beta_{2}+k_{d} \beta_{1}+k_{p}\right) s\right\}} . \\
& F(s)=\frac{\left(k_{p} \beta_{1}+k_{d} \beta_{2}+\beta_{3}\right) s^{2}+\left(k_{p} \beta_{2}+k_{d} \beta_{3}\right) s+k_{p} \beta_{3}}{k_{p}\left(s^{3}+\beta_{1} s^{2}+\beta_{2} s+\beta_{3}\right)} . \\
& G_{p}(s)=\frac{L s^{2}+g}{M L s^{4}+D L s^{3}+\left((M+m) g-k_{p}\right) s^{2}+D g s} .
\end{aligned}
$$

The poles of the closed-loop system are all located in the left half plane of the s plane, which indicates the system is stable.

\section{Performance of ESO and Feedforward Controller Design}

From Eq.17 :

$$
\left\{\begin{array}{l}
z_{1}=\frac{\beta_{1} s^{2}+\beta_{2} s+\beta_{3}}{s^{3}+\beta_{1} s^{2}+\beta_{2} s+\beta_{3}} y+\frac{b_{0} s}{s^{3}+\beta_{1} s^{2}+\beta_{2} s+\beta_{3}} u \\
z_{2}=\frac{\beta_{2} s+\beta_{3}}{s^{3}+\beta_{1} s^{2}+\beta_{2} s+\beta_{3}} y+\frac{b_{0}\left(s+\beta_{1}\right) s}{s^{3}+\beta_{1} s^{2}+\beta_{2} s+\beta_{3}} u \\
z_{3}=\frac{\beta_{3} s^{2}}{s^{3}+\beta_{1} s^{2}+\beta_{2} s+\beta_{3}} y-\frac{b_{0} \beta_{3}}{s^{3}+\beta_{1} s^{2}+\beta_{2} s+\beta_{3}} u
\end{array}\right.
$$

The estimated errors are:

$$
\left\{\begin{array}{l}
E_{1}=z_{1}-y=-\frac{s^{3}}{s^{3}+\beta_{1} s^{2}+\beta_{2} s+\beta_{3}} y+\frac{s}{s^{3}+\beta_{1} s^{2}+\beta_{2} s+\beta_{3}} b_{0} u \\
E_{2}=z_{2}-\dot{y}=-\frac{\left(s+\beta_{1}\right) s^{3}}{s^{3}+\beta_{1} s^{2}+\beta_{2} s+\beta_{3}} y+\frac{s}{s^{3}+\beta_{1} s^{2}+\beta_{2} s+\beta_{3}} b_{0} u \\
E_{3}=z_{3}-f=z_{3}-\ddot{y}-b_{0} u=b_{0}\left(1-\frac{\beta_{3}}{s^{3}+\beta_{1} s^{2}+\beta_{2} s+\beta_{3}}\right) u-\left(1-\frac{\beta_{3}}{s^{3}+\beta_{1} s^{2}+\beta_{2} s+\beta_{3}}\right) s^{2} y
\end{array}\right.
$$

From Eq.14 and Eq.15:

$y=\frac{1}{s^{2}}\left(u_{0}+f-z_{3}\right)=\frac{1}{s^{2}}\left(u_{0}+E_{3}\right)$.

Typically, $y$ and $u$ are taken as step signals with amplitude of $\mathrm{K}$, the steady state errors are: 


$$
\left\{\begin{array}{l}
e_{s s 1}=\lim _{t \rightarrow \infty} e(t)=\lim _{s \rightarrow 0} s E_{1}(s)=0 \\
e_{s s 2}=\lim _{t \rightarrow \infty} e(t)=\lim _{s \rightarrow 0} s E_{2}(s)=0 \\
e_{s s 3}=\lim _{t \rightarrow \infty} e(t)=\lim _{s \rightarrow 0} s E_{3}(s)=0
\end{array}\right.
$$

In the above case, ESO converges to estimate the system state variables and generalized perturbation. Then the system can be transformed into a series of integral system. Literature [10] stated that if $\dot{f}$ is bounded, There always exists an ESO that the estimated error is bounded. The system will be partly transformed into cascaded integrators. We can choose the feedforward controller as $\ddot{r}$, but due to the estimated error, it is often excessive, so $0.4 \ddot{r}$ is chosen as a feedforward controller.

\section{Simulation Results and Comparison}

In the simulation, we compare the simulation results of improved ADRC control with PID control which is widely used in engineering.

Two PID controllers are used to control the position of the trolley and swing angle respectively. PID parameters for position control are set as: $K_{p}=50, K_{I}=30, K_{D}=40$. PID parameters for angle control are set as: $K_{p}=40, K_{I}=10, K_{D}=20$ [9]. Set the parameters of the system as follows: $M=15 \mathrm{~kg}$, $b_{0}=0.084$, coefficient of friction $D=0.3$, observer bandwidth $\omega_{0}=15$, system bandwidth $\omega_{c}=2$, the proportional controller gain $k_{p}=40$. When modeling, $L=0.5, m=5$.

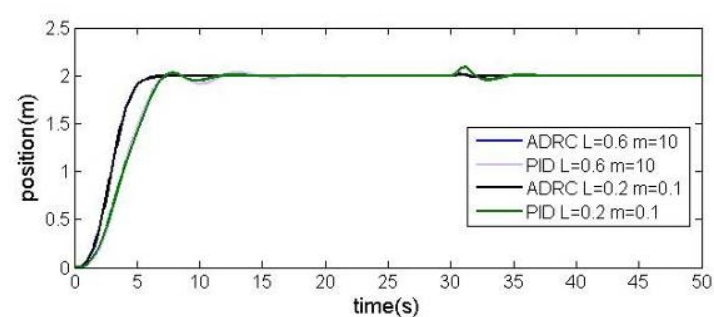

(a)

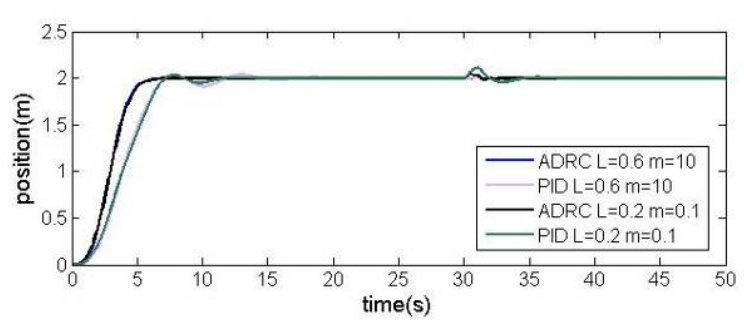

(b)

Fig.5: (a)Position of the trolley; (b) Position of the load.

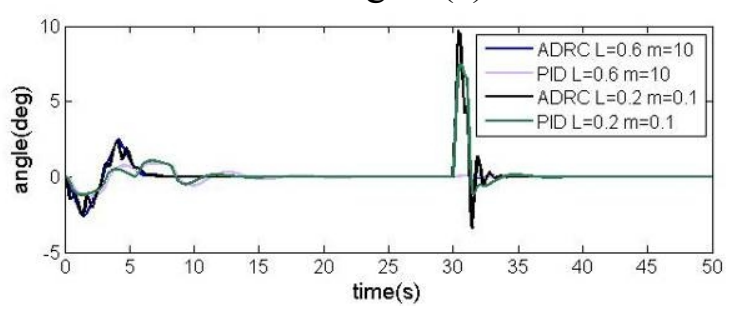

(a)

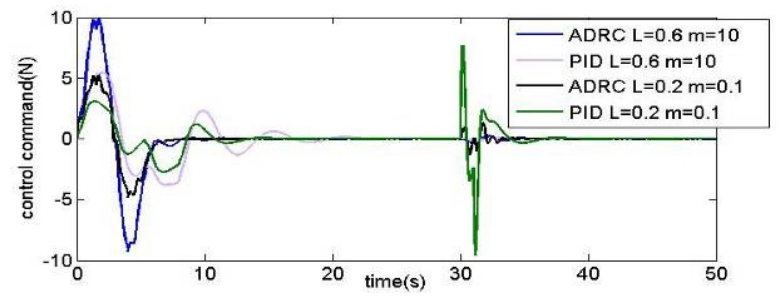

(b)

Fig.6: (a) Angle of the load; (b) control command with disturbance.

Both the length of the rope and the weight of the load are changed in the simulation test, the other parameters remain unchanged. The wind disturbance is exerted at $30 \mathrm{~s}$, the simulation results are as Fig.5 and Fig.6.

The simulation shows that the trolley and the load are affected by the wind disturbance. Though the load is more influenced when it's too light, the trolley is less influenced when using the improved ADRC. Changes in position of the trolley and load which are controlled by ADRC controller are significantly lower than PID control. The improved ADRC can quickly eliminate the disturbance of the trolley and the load. Considering the extreme case $m=0.1$, the proposed controller can also eliminate the swing angle quickly. So ADRC-P with feedforward controller has good dynamic and steady-state performance. The proposed method has a more excellent performance is because that ADRC has a natural decoupling characteristics. It treats the shock of the load and the variety of system parameters as a total disturbance, use an ESO to estimate it in real time, and then cancel it in the 
control law. At the same time, the number of adjusting parameters of the proposed solution is significantly less than PID, which can save more time and resources.

\section{Conclusion}

In this paper, an ADRC control algorithm with a combination of feedforward and proportional controller for a nonlinear, strongly coupled and under-actuated system overhead crane system is presented. The simulation results show that this controller can overcome some model uncertainties, resist to external disturbances, and achieve the goal of accurate positioning of the trolley and load anti-swing. The improved ADRC this paper proposed is effective in the anti-swing and it has a better performance of quickness, so it makes the crane system robust and practical.

\section{References}

[1] Sorensen K L. A command feedback and command shaping controller for improving position and reducing cable sway in cranes [D]. Georgia: School of Mechanical Engineering Georgia Institute of Technology, 2005.

[2] Y. Yoshida, H. Tabata. Visual Feedback Control of an Overhead Crane and Its Combination with Time-Optimal Control [C]. Proceedings of the 2008 IEEE/ASME International Conference on Advanced Intelligent Mechatronics. Xi'an, China, 2008: 1114-1119.

[3] Lee Ho-Hoon, Cho Sung Kun. A New Fuzzy-Logic Anti-Sway Control for Industrial Three-Dimensional Overhead Cranes [C]. Proceeding of the 2001 IEEE International Conference on Robotic\&Automation, 2001 (5): 2956-2961.

[4] W. Wang, J. Yi, D. Liu. Design of a stable sliding mode controller for a class of second-order underactuated systems [J]. IEEE Control Theory and Applications, 2004,151 (6): 683-690.

[5] J. Han. ADRC Control technologies to compensate the estimated uncertainties [M]. Beijing: National Defence Industry Press, 2009.

[6] Hanafy M. Omar, Control of Gantry and Tower Cranes [D]. Virginia: Virginia Polytechnic Institute and State University, 2003.

[7] King Shyang Sien, Command Shaping Control of a Crane Syatem [D]. Faculty of Electrical Engineering University Technology Malaysia, 2006.

[8] G. Tian, Z. Gao. Frequency Response Analysis of Active Disturbance Rejection Based Control System [C].16 ${ }^{\text {th }}$ IEEE International Conference on Control Applications, 2007: 1595-1599.

[9] M. Wang. PID adjustment and parameter setting [J]. Science and Technology Innovation Herald. 2009 (31): 57.

[10]Q. Zheng, Linda Q. Gao, Z. Gao, On Validation of Extended State Observer Through Analysis and Experimentation [J]. Journal of Dynamic Systems, Measurement, and Control. 2012 (134): 024505. 